\title{
Higher lung accumulation of intravenously injected organic nanotubes
}

\author{
This article was published in the following Dove Press journal: \\ International Journal of Nanomedicine \\ 17 January 2013 \\ Number of times this article has been viewed
}

\author{
Yoshie Maitani' \\ Yuri Nakamura' \\ Masao Kon' \\ Emi Sanada' \\ Kae Sumiyoshi' \\ Natsuki Fujine' \\ Masumi Asakawa ${ }^{2}$ \\ Masaki Kogiso ${ }^{2}$ \\ Toshimi Shimizu² \\ 'Institute of Medicinal Chemistry, \\ Hoshi University, Tokyo, Japan; \\ ${ }^{2}$ Nanotube Research Center (NTRC), \\ National Institute of Advanced \\ Industrial Science and Technology \\ (AIST), Tsukuba, Japan
}

Correspondence: Yoshie Maitani Institute of Medicinal Chemistry, Hoshi University, Ebara 2-4-4I, Shinagawa-ku, Tokyo I42-850I, Japan Tel +8I 354985048

Fax +8I 354985048

Email yoshie@hoshi.ac.jp
Abstract: The size and shape of intravenously injected particles can affect their biodistribution and is of importance for the development of particulated drug carrier systems. In this study, organic nanotubes (ONTs) with a carboxyl group at the surface, a length of approximately $2 \mu \mathrm{m}$ and outer diameter of 70-90 nm, were injected intravenously into tumor-bearing mice. To use ONTs as drug carriers, the biodistribution in selected organs of ONTs postinjection was examined using irinotecan, as an entrapped water-soluble marker inside ONTs, and gadolinium-chelated ONT, as an ONT marker, and compared with that of a $3 \mu \mathrm{m}$ fluorescently labeled spherical microparticle which was similar size to the length of ONTs. It was found that for irinotecan, its active metabolite and gadolinium-chelated ONTs were highly accumulated in the lung, but to a lower level in the liver and spleen. On the other hand, microparticles deposited less in the lung and more highly in the liver. Moreover, histologic examination showed ONTs distributed more in lung tissues in part, whereas microparticles were present in blood vessels postinjection. These preliminary results support the notion of using negatively charged ONTs as intravascular carriers to maximize accumulation in the lung whilst reducing sequestration by the liver and spleen. This finding suggested that ONTs are potential carriers for lung-targeting drug delivery.

Keywords: organic nanotube, lung, biodistribution, microparticle, particle shape

\section{Introduction}

Biodistributions of spherical particles such as liposomes and polymer micelles as drug carriers, have been investigated after injection since intravenously injected particles have the potential to enable the targeted delivery of therapeutic agents. ${ }^{1}$ With regard to particle size, smaller nonbiodegradable rigid polystyrene microparticles (MPs) $(<4 \mu \mathrm{m})$ pass through the lung and become entrapped in the organs of the reticuloendothelial system (RES), such as the liver and spleen ${ }^{2,3}$ and larger MPs are accumulated in the lung. ${ }^{3}$ For surface properties, it is well established that modification with polyethylene glycol (PEG) chains helps nanosized particles to evade RES uptake and thus remain in the circulation for a longer time. Non-PEGylated, nanosized liposomes consistently tend to accumulate in RES. ${ }^{4}$

The effects of shape on biodistribution have received little attention. Recently, nonspherical particles such as cylindrical particles and rod-like particles have been investigated. ${ }^{5}$ Injected single-walled carbon nanotubes (SWCNT) with a diameter of $\sim 1.2 \mathrm{~nm}$ and length of 0.1 to $1 \mu \mathrm{m}$ were rapidly excreted through the glomeruli of the kidneys, and a transitional accumulation was detected in the liver and spleen. ${ }^{6}$ Polymer micelle assemblies known as filomicelles with $\sim 8 \mu \mathrm{m}$ length persisted 
in the circulation for up to 1 week after intravenous injection and the liver and spleen also dominates the clearance of filomicelles. ${ }^{7}$ On the other hand, silicon-based particles with discoidal shape and a diameter of $1.6 \mu \mathrm{m}$ were reported to accumulate highly in the lung. ${ }^{8}$

Organic nanotubes (ONTs) have a cylindrical structure that self-assembles from amphiphilic molecules in aqueous media. ONTs have an inner diameter of 10 to $200 \mathrm{~nm}$, an outer diameter of 40 to $1000 \mathrm{~nm}$, and a length of approximately $2 \mu \mathrm{m}$ to several hundred micrometers. Furthermore, these dimensions are controllable by rationalizing the molecular structure of the amphiphilic molecules. ${ }^{9,10}$ ONTs hold promise for applications as target delivery carriers in the field of nanomedicine. Therefore, when intravenously administered in vivo, their biodistribution needs to be fully characterized.

Previously we have reported that ONTs with a carboxyl group on the surface and an inner diameter of 30-40 nm, outer diameter of 70-90 nm, and a length of approximately $2 \mu \mathrm{m}$ were taken up in nonphagocytic mouse colon adenocarcinoma 26 cell line (C26) cells in vitro. ${ }^{11}$ Here, we focused on drug delivery application of ONTs in vivo.

In this study, using the anticancer drugs, irinotecan (CPT-11) and gadolinium (Gd) as tracers for the ONT carrier, the biodistribution of ONTs loaded with CPT-11 and Gd-chelated ONT was examined. Similar-sized spherical particles to the length of ONTs, $3 \mu \mathrm{m}$ MPs was used as a control. The biodistribution of various sized MPs has been reported. ${ }^{3}$ Similar-sized spherical particles to the diameter of ONTs, approximately $100 \mathrm{~nm}$ non-PEGylated liposomes and MPs are already known to be accumulated in RES. ${ }^{4}$ More than $4 \mu \mathrm{m}$-sized MPs accumulated in the lung in rats, ${ }^{3}$ and therefore $3 \mu \mathrm{m}$-sized MPs may accumulate in lung of mice. Here, we present a preliminary analysis of how the shape of the particles can affect their accumulation in major organs after intravenous injection. The biodistribution of ONTs was studied in tumor-bearing mice, compared with MPs of $3 \mu \mathrm{m}$ in size.

\section{Material and methods}

\section{Materials, zeta-potential,}

\section{and image measurement}

CPT-11 was a kind gift from Yakult Co, Ltd (Tokyo, Japan). Doxorubicin (DXR) hydrochloride was purchased from Wako Pure Chemical Industries (Osaka, Japan). Fluorescent, internally fluorescein isothiocyanate (FITC)-labeled, polystyrene MPs of $3 \mu \mathrm{m}$ size were purchased from Polysciences Inc (Warrington, PA). 2-Ethoxyethyl acetate was purchased from
Tokyo Chemical Ind. Co, Ltd (Tokyo, Japan). ONTs are easily formed by evaporating methanolic solutions of glycylglycine and myristic acid (Figure 1, compound 1). ${ }^{11}$ ONTs were prepared at the Nanotube Research Center (NTRC), National Institute of Advanced Industrial Science and Technology (AIST) (Tsukuba, Japan). ${ }^{12} \mathrm{Gd}^{3}$-chelated ONT (Gd-ONT), which contained $12.6 \mathrm{wt} \%$ of Gd (III) ions, was also formed from $\mathrm{GdCl}_{3}$ and compound 1 as described previously. ${ }^{12}$ Other reagents were of analytical or high-pressure liquid chromatography (HPLC) grade. Figure 1 shows schematic illustrations of ONT and Gd-chelated ONT (Gd-ONT). Figure 2 shows fieldemission scanning electron microscopy (FESEM) images of ONTs. The zeta-potential of the ground ONT was measured in water at $25^{\circ} \mathrm{C}$ using a Photal ELS-Z2 zeta-potential analyzer (Otsuka Electronics Co, Ltd, Osaka, Japan). FESEM image was taken by using a Hitachi S-4800 field emission electron microscope at $15 \mathrm{kV}$ (Hitachi High-Tech, Tokyo, Japan).

\section{Preparation of ONTs loaded with CPT-II and drug release}

To prepare ONTs loaded with CPT-11 (CPT-11/ONT), ground ONT ( $\sim 2 \mathrm{mg}$, approximate length $\sim 2 \mu \mathrm{m})$ and CPT-11 aqueous solutions $(3,5,7.5 \mathrm{mg} / \mathrm{mL}, 100 \mu \mathrm{L}$ of $\mathrm{pH} 4$ or $\mathrm{pH} 6$, deionized and MilliQ water [water] adjusted using $1 \mathrm{~N} \mathrm{HCl}$ ) were mixed, then vortexed for 10 minutes, and sonicated. Then, $900 \mu \mathrm{L}$ of phosphate-buffered saline (PBS, $\mathrm{pH}$ 7.4) was added to the mixture of CPT-11 and ONTs as reported previously. ${ }^{11}$ To measure the entrapment efficiency of CPT-11 into ONTs, the mixing weight ratio of ONTs and CPT-11 and $\mathrm{pH}$ of the medium were changed. After mixing, CPT-11 and ONT in the medium were centrifuged $(20,400 \mathrm{~g}$, $20^{\circ} \mathrm{C}, 30$ minutes), the precipitate was washed with water as CPT-11/ONT, and the CPT-11 concentration in the supernatant was analyzed by HPLC. ${ }^{13}$ CPT-11 was detected by
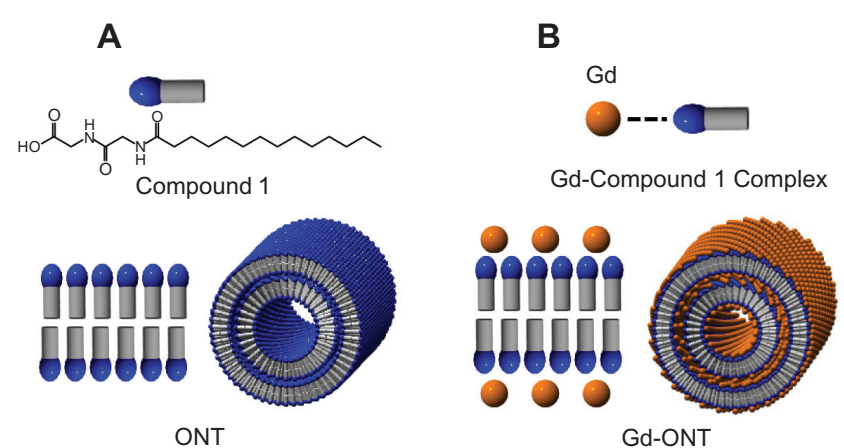

Figure I Chemical structure of compound I that forms the structure of ONTs and schematic illustrations of ONT (A) and Gd-chelated ONT (Gd-ONT) (B).

Note: Compound I consists of glycylglycine and myristic acid (molecular weight 342). Abbreviations: Gd, gadolinium; ONTs, organic nanotubes. 


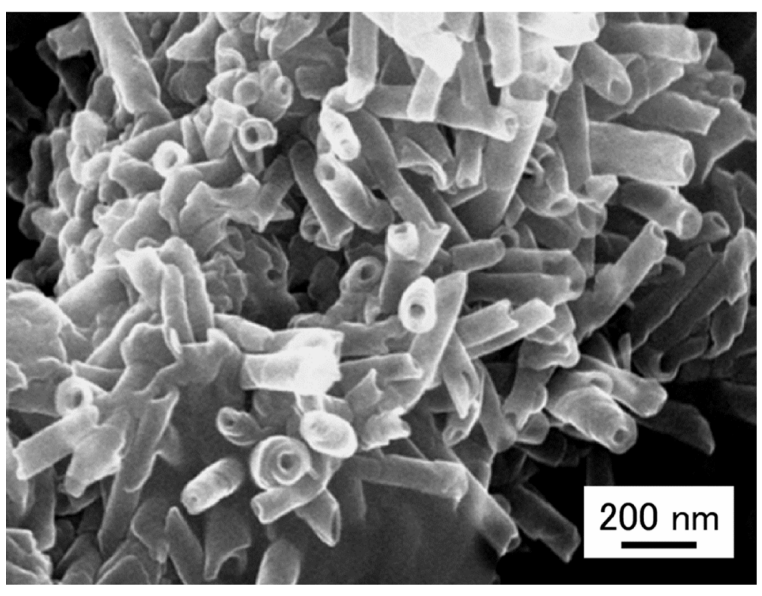

Figure 2 Field-emission scanning electron microscopy (FESEM) images of ONTs. Abbreviation: ONTs, organic nanotubes.

excitation and emission wavelengths of 485 and $535 \mathrm{~nm}$, respectively. Then, the loading amount and loading efficiency were calculated using the following equations:

Loading amount $(\mu \mathrm{g} / \mathrm{mg})$ $=\frac{\text { Added drug }(\mu \mathrm{g})-\text { Remained drug in supernatant }(\mu \mathrm{g})}{\text { ONT amount }(\mathrm{mg})}$

Loading efficiency (\%)

$$
\begin{aligned}
& =\frac{\text { Added drug }(\mu \mathrm{g})-\text { Remained drug in supernatant }(\mu \mathrm{g})}{\text { Added drug }(\mu \mathrm{g})} \\
& \times 100
\end{aligned}
$$

The release of CPT-11 from CPT-11/ONT in a dialysis tube was measured using seamless cellulose tube membranes (Viskase Sales Corp., Chicago, IL, molecular weight cut-off $[\mathrm{MWCO}]=2000$ ). The sample volume in the dialysis tube was $1 \mathrm{~mL}$ with $1 \mathrm{mg}$ ONT as the initial concentration, and the sink volume was $100 \mathrm{~mL}$ of PBS at $\mathrm{pH} 7.4$ and $\mathrm{pH} 5.5$ at $37^{\circ} \mathrm{C}$ as reported previously. ${ }^{14}$ The CPT- 11 concentration was analyzed using HPLC as described below.

\section{Animal experiments}

A mouse colon adenocarcinoma 26 cell line (C26) was obtained from the Cell Resource Center for Biomedical Research, Tohoku University (Miyagi, Japan). The cells were cultured in RPMI-1640 medium containing 10\% heatinactivated fetal bovine serum (Invitrogen Corp. Carlsbad, $\mathrm{CA}$ ) and $100 \mu \mathrm{g} / \mathrm{mL}$ kanamycin sulfate in a humidified atmosphere containing $5 \% \mathrm{CO}_{2}$ at $37^{\circ} \mathrm{C}$. Five-week-old ddY female mice and CDF1 female mice were purchased from Sankyo Labo Service Corporation (Tokyo, Japan). All animal experiments were carried out in accordance with the guidelines of the Guiding Principles for the Care and Use of
Laboratory Animals of Hoshi University. To generate C26 tumors, $1 \times 10^{6}$ cells suspended in $100 \mu \mathrm{L}$ of RPMI medium were inoculated subcutaneously into the flank of female CDF1 mice (5 weeks of age; 20-22 g).

\section{Biodistribution analysis}

The tumor volume was calculated using the formula; tumor volume $=0.5 \times \mathrm{a} \times \mathrm{b}^{2}$, where $\mathrm{a}$ and $\mathrm{b}$ are the larger and smaller diameters, respectively. When the average volume of the tumors reached $100-150 \mathrm{~mm}^{3}$, free CPT-11 in saline, CPT-11/ONT in saline, and Gd-ONT in saline containing $0.1 \%$ Tween 80 were intravenously injected via the lateral tail veins. Free CPT-11 at a dose of $4 \mathrm{mg}$ CPT-11/kg, CPT-11/ONT at a dose of $100 \mathrm{mg}$ ONT/kg corresponding to a concentration of $2-3.5 \mathrm{mg} \mathrm{CPT}-11 / \mathrm{kg}$, and Gd-ONT at a dose of $50 \mathrm{mg}$ ONT $/ \mathrm{kg}$ corresponding to a concentration of $6.3 \mathrm{mg} \mathrm{Gd} / \mathrm{kg}$ were applied. In a preliminary experiment, it was confirmed that a dose of $100 \mathrm{mg}$ ONT/ $\mathrm{kg}$ was safe. ${ }^{11}$ Fluorescent MPs were washed using distilled water before being suspended in saline containing $0.1 \%$ Tween $80(20 \mathrm{mg}$ $\mathrm{MPs} / \mathrm{mL}$ ). MPs were fully suspended in the solution by sonicating and vortexing immediately prior to intravenous administration at a dose of $25 \mathrm{mg} / \mathrm{kg}$. At predetermined time points, blood for CPT-11/ONT and Gd-ONT was collected using a syringe and centrifuged to obtain serum at $1500 \mathrm{~g}$ for 30 minutes. CPT-11 and SN-38 in serum were extracted with the addition of an equal volume of cold acidic methanol. The mixture was vortexed for 10 seconds and incubated at $-80^{\circ} \mathrm{C}$ for more than 5 hours until analysis. The liver, spleen, kidneys, heart, lung, and tumor were removed, rinsed in saline, weighed and frozen at $-80^{\circ} \mathrm{C}$.

\section{HPLC apparatus and conditions}

After thawing, blood samples were centrifuged at $100,000 \mathrm{~g}$ for 30 minutes at $4^{\circ} \mathrm{C}$ by ultracentrifugation (CS120 GXL; Hitachi) to remove aggregated protein as reported previously. ${ }^{13}$ The supernatant was applied to a TSKgel ODS80Ts QA $5 \mu \mathrm{m}$ column $(4.6 \mathrm{~mm}$ I.D. $\times 25 \mathrm{~cm}$, Tosoh, Co, Ltd, Tokyo, Japan) equilibrated in $75 \mathrm{mM}$ ammonium acetate, $35 \%$ acetonitrile, $\mathrm{pH} 4.0$, at a flow rate of $1 \mathrm{~mL} /$ minute. CPT-11 and SN-38 were detected using an HPLC system (Shimadzu Co, Kyoto, Japan) composed of an LC-10 AS pump, an SIL-10A autoinjector and an RF-10 AXL fluorescence detector at an excitation wavelength of $375 \mathrm{~nm}$ and an emission wavelength of $530 \mathrm{~nm}$. The tumor, liver, spleen, kidney, and lung were homogenized in PBS. CPT-11 and SN-38 were extracted with cold acidic methanol and analyzed by the HPLC system as described above. The drug dose $\% / \mathrm{mL}$ 
serum and $g$ tissue were calculated by dividing the amount of drug per total serum volume $(\mathrm{mL})$ and per total tissue weight $(\mathrm{g})$, respectively, as reported previously. ${ }^{13}$

\section{Analysis of Gd amounts in the organs}

The serum Gd content was measured by means of inductively coupled plasma mass spectrometry (ICP-MS) with an SPS7800 apparatus (SII NanoTechnology Inc, Tokyo, Japan). For the determination of Gd ion content in the tissue, saline was added to the tissues, followed by an addition of nitric acid (conc 70\%) and sulfuric acid (conc 98\%). Then, the mixture was heated. A saturated aqueous oxoammonium solution was added to the yellow mixture and heated again. The resulting pale yellow mixture was diluted with saline. The supernatant was collected, and its Gd content was measured by means of ICP-MS. Urine was collected $24 \mathrm{~h}$ postinjection, and its Gd content was also measured by means of ICP-MS.

\section{Quantitation of MPs}

The analytical method for quantification of MPs in organs was modified as follows. ${ }^{3,15}$ Briefly, the organ samples were digested using approximately $8 \mathrm{~mL}$ ethanolic $\mathrm{KOH}$ at $50^{\circ} \mathrm{C}$ for 48 hours with shaking. After centrifugation, the supernatant was discarded, then the digested samples were washed with approximate $8 \mathrm{~mL} \mathrm{1 \%}$ Triton X-100, and finally washed once with $7 \mathrm{~mL}$ PBS. After centrifugation, the supernatant was discarded, then $200 \mu \mathrm{L}$ water and $3 \mathrm{~mL}$ 2-ethoxyethyl acetate were added to each sample, and the samples were mixed thoroughly before being stored in the dark at room temperature for 5 days. After centrifugation, the supernatant was examined for fluorescent MPs and analyzed in triplicate using a fluorescence spectrophotometer (F-2700; Hitachi) with an excitation wavelength of $430 \mathrm{~nm}$ and emission wavelength at $510 \mathrm{~nm}$.

\section{Distribution in lung and histology studies}

To observe the lung distribution of ONT, ONTs loaded with DXR (DXR/ONT) as a fluorescent marker was prepared as reported previously. ${ }^{11} \mathrm{DXR} / \mathrm{ONT}$ was the amount loaded in DXR $44.2 \mu \mathrm{g} / \mathrm{mg}$ ONT. ${ }^{11}$ DXR/ONT and MPs were injected at a dose of $50 \mathrm{mg} \mathrm{ONT} / \mathrm{kg}$ and $25 \mathrm{mg} \mathrm{MP} / \mathrm{kg}$. For detection of lung vessels with blood flow, fluorescent DNA-binding dye Hoechst 33342 was injected at $10 \mathrm{mg} / \mathrm{kg}$ into the tail vein 1 minute before sacrifice. At 3 hours after a single injection, a portion of the lung were collected and prepared as $6-\mu \mathrm{m}$ frozen sections. Tissue sections were examined using an inverted microscope, ECRIPS TS100 (Nikon, Tokyo, Japan) as reported previously. ${ }^{16}$
For histological inspection, upon euthanasia after the administration of saline, ONTs $(50 \mathrm{mg} / \mathrm{kg})$ and MP $(25 \mathrm{mg} / \mathrm{kg})$, the lung was collected. The tissue was immediately frozen in dry ice. Frozen $6-\mu \mathrm{m}$ sections were cut. The tissue slides were stained using hematoxylin and eosin (H\&E) dye, and observed histopathologically using a microscope to check for any possible tissue damage.

\section{Statistical analysis}

The results are expressed as the mean \pm standard deviation. Statistical comparisons were performed using Student's $t$-test. $P$ values less than 0.05 were considered significant.

\section{Results \\ CPT-I I/ONT characterization in loading and release of CPT-I I}

First, to examine the effect of $\mathrm{pH}$ on the loading of CPT-11 into ONTs, CPT-11 was dissolved in water at $\mathrm{pH} 4$ or 6. CPT-11 loading efficiency into ONTs at a weight ratio of CPT-11:ONT of 0.25 significantly increased to $83 \pm 4 \mu \mathrm{g}$ CPT-11/mg ONT when the $\mathrm{pH}$ was reduced from 6 to 4 $(P<0.05)$ (Figure 3A).

Next, the effect of the weight ratio of CPT-11 to ONT on CPT-11 loading was examined at $\mathrm{pH} 4$ (Figure 3B). As the CPT-11:ONT ratio increased, the CPT-11 loading amount significantly increased to $115 \pm 24 \mu \mathrm{g} / \mathrm{mg}$ ONT $(P<0.05)$, however, loading efficiency was decreased slightly. From these findings, a weight ratio of CPT-11:ONT of 0.25 in water at $\mathrm{pH} 4$ was selected as the drug-loading condition in the following experiment because of the adequate loading efficiency $(16.7 \%$, ie, the weight ratio of CPT-11:ONT $=1: 25)$ and the ease of handling. CPT-11/ONT was confirmed to retain the nanotube structure using a microscope because the nonspherical size could not be measured using dynamic light scattering. The preparation of CPT-11/ONT indicated
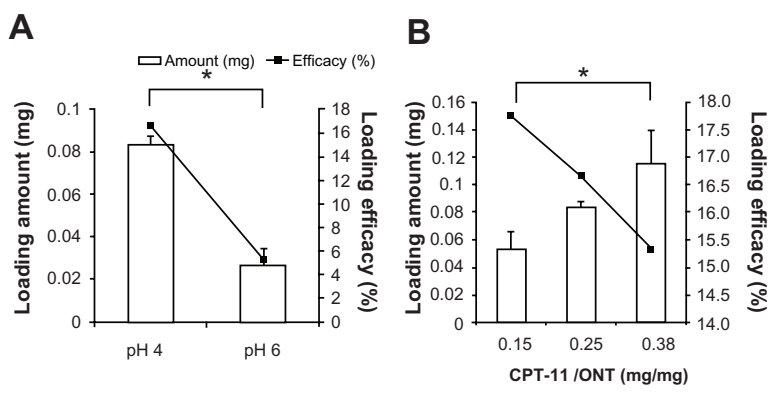

Figure 3 Effect of $\mathrm{pH}$ and ratio of CPT-II:ONT (w:w) on loading amount and loading efficiency of CPT-II into ONTs at a CPT-II:ONT weight ratio of 0.25 (A) and at $\mathrm{pH} 4.0$ (B) at room temperature.

Note: Each value represents mean \pm SD $(n=3)$.

Abbreviations: ONTs, organic nanotubes; SD, standard deviation. 
that the loading efficiency of CPT-11 increased with the decreasing $\mathrm{pH}$ of the medium.

CPT-11 released from the CPT-11/ONT prepared as described previously was evaluated for 24 hours in PBS at $\mathrm{pH} 7.4$ and $\mathrm{pH} 5.5$ at $37^{\circ} \mathrm{C}$. The release of CPT-11 from ONTs at pH 7.4 was $\sim 60 \%$ over 1 day higher, which was greater than that at $\mathrm{pH} 5.5$ (Figure 4). This may be caused by a reduction in the electrostatic interaction between CPT-11 and ONT because the dissociation of weak basic CPT-11 at higher $\mathrm{pH}$ was reduced.

\section{Biodistribution of ONTs and MPs}

The biodistribution of ONTs was examined using CPT-11, as an entrapped water soluble marker inside ONTs, and GdONT, as an ONT marker (Figure 1). Compound 1 catches metal ion very strongly; therefore Gd ion is scarcely released from Gd-ONT. ${ }^{12}$ The biodistribution of Gd corresponds to that of Gd-ONT. To evaluate the biodistribution of ONTs, free CPT-11, CPT-11/ONT and Gd-ONT were injected intravenously into mice bearing C26 tumors. At 24 hours postinjection, CPT-11 from CPT-11/ONT was largely distributed in the lung and in the spleen compared with free CPT-11 (Figure 5A, $P<0.05$ ). Similar to CPT-11, the active metabolite, SN-38, was highly accumulated in the lung as well as liver, indicating the high lung distribution of CPT-11/ ONT (Figure 5B) because liposomal SN-38 did not exhibit such a specific lung distribution. ${ }^{13}$ CPT-11 from CPT-11/ONT was detected in serum and tumors in higher levels than free CPT-11 at 24 hours postinjection, suggesting that CPT-11/ ONT could circulate in the blood for a long period.

In common with CPT-11/ONT, Gd-ONT was also largely distributed in the lung (Figure 6). The lung accumulation of

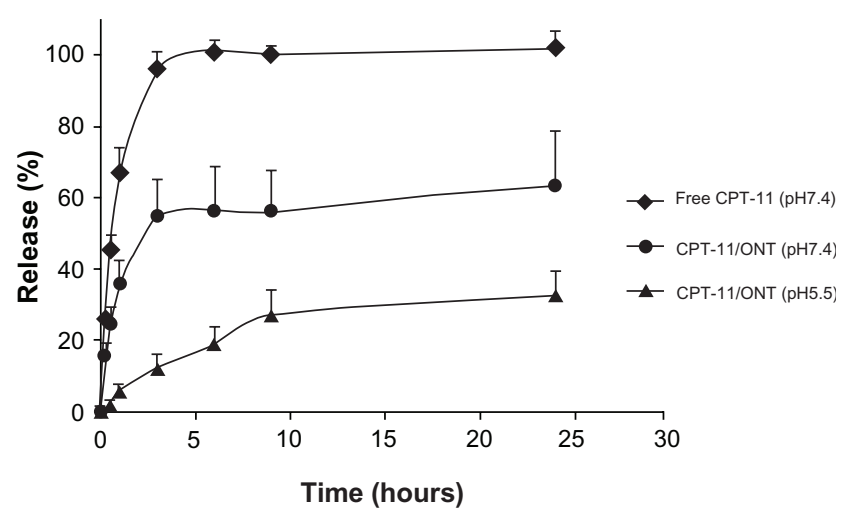

Figure 4 CPT-II release from free CPT-II and CPT-I I/ONT in a dialysis tube in $\mathrm{PBS}$ at $\mathrm{pH} 5.5$ and $\mathrm{pH} 7.4$ at $37^{\circ} \mathrm{C}$.

Note: Each value represents mean \pm SD $(n=3)$.

Abbreviations: ONTs, organic nanotubes; PBS, phosphate-buffered saline; $\mathrm{SD}$, standard deviation.
Gd-ONT decreased to $54.2 \% \pm 10.9 \%, 42.9 \% \pm 2.28 \%$ and $24.9 \% \pm 10.4 \%$ of the dose/g of lung at 3, 24, and 48 hours postinjection, in a time-dependent manner. The liver and spleen accumulation of Gd-ONT was $7.7 \% \pm 3.8 \%$ and $4.9 \% \pm 2.6 \%$ of dose/g of tissue, respectively at 3 hours postinjection, which increased slightly at 24 hours, and decreased at 48 hours. Gd-ONT was detected in serum 24 hours postinjection, suggesting that ONT circulated in the blood for at least 24 hours postinjection because Gd-DTPA was very rapidly cleared from the bloodstream with a half-life in the order of minutes. ${ }^{17}$ The difference of each accumulation of CPT-11 and Gd-ONT dose percentage in tissues may be due to that of entrapped drug and carriers. Dose of CPT-11 from limited dose of ONTs was low, and CPT-11/ONT may rapidly release CPT-11 into the circulation postinjection.

To examine the effect of shape on the biodistribution of ONTs, $3 \mu \mathrm{m}$ MP was injected intravenously and the biodistribution of MPs was compared with that of ONTs. The biodistribution of MPs exhibited high accumulation of $42.4 \% \pm 1.35 \% / \mathrm{g}$ liver and $56.5 \% \pm 14.6 \% / \mathrm{g}$ spleen and low accumulation with $4.96 \% \pm 0.74 \% / \mathrm{g}$ lung $(0.8 \% / \mathrm{lung})$ at 24 hours postinjection (Figure 7). This finding in mice was consistent with a previous report that $3 \mu \mathrm{m}$ MP was accumulated at a level of less than $10 \%$ in the lung, but higher in the liver and spleen at 6-48 hours postinjection in rats. ${ }^{3}$

To gain further insight into the localization of ONTs in the lung, histological analyses were performed 3 hours after injection and compared with that of MPs (Figure 8). For this purpose, DXR/ONT was used as a fluorescent marker of ONTs. Red dots, green dots and blue fluorescence represent DXR/ONT, FITC-labeled MPs and blood vessels stained by Hoechst 33342, respectively. Using fluorescence microscopy analyses, autofluorescence of lung tissues postinjection of saline was observed, but red dots of DXR/ONT were found outside blood vessels in part whereas green dots of MPs were exclusively found within the vessel lumen or associated with the endothelial cells. In the saline control groups, such red dots of DXR/ONT and green dots of MPs were not observed.

Because ONTs were highly distributed in lung tissues at 3 hours postinjection, to observe the integrity of the lung, histological lung tissue sections were examined. No histological changes or any signs of acute toxicological effects were observed in the lung tissues postinjection of ONT compared with the saline control (Figure 9).

\section{Discussion}

To use ONTs as drug carriers, the biodistribution of ONTs postinjection was examined using CPT-11, as an entrapped 


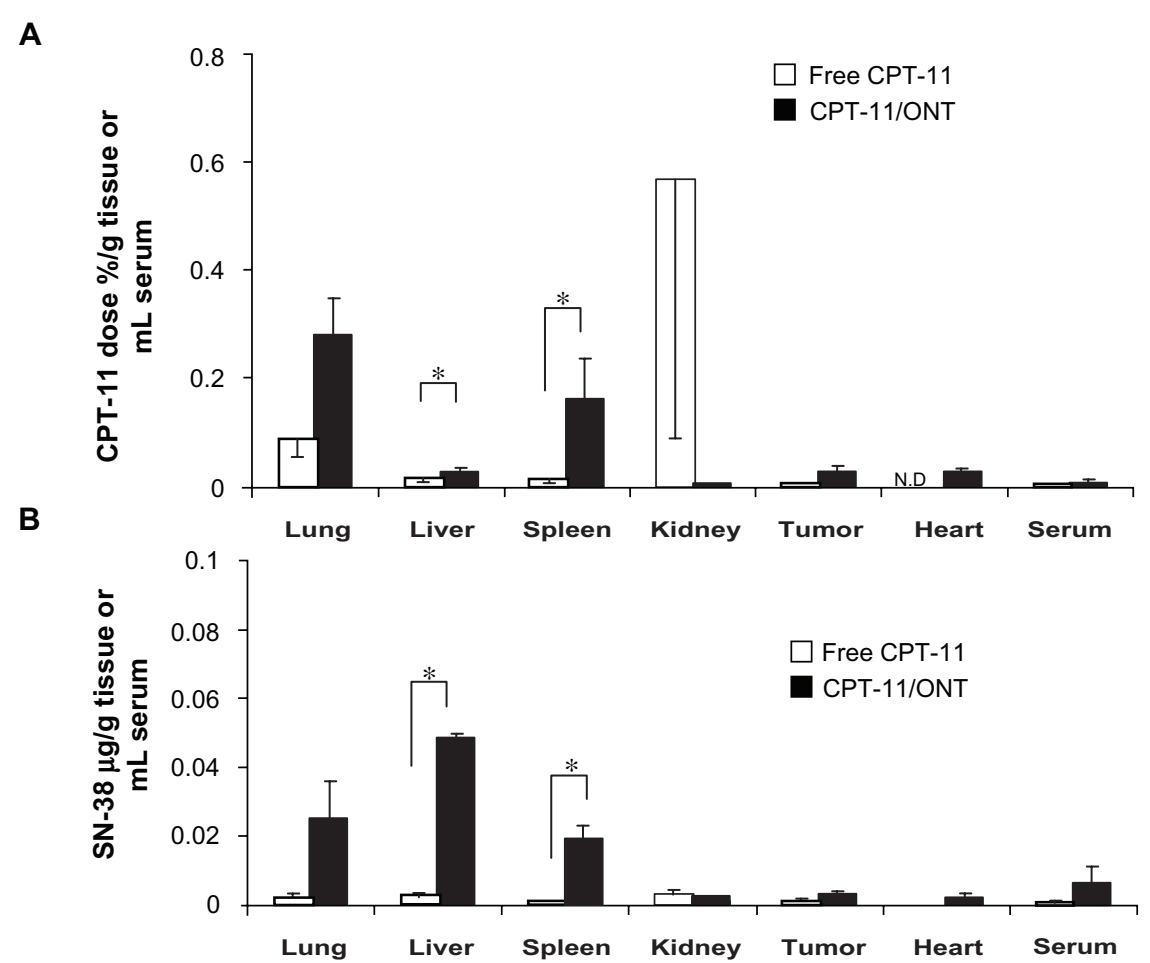

Figure 5 Biodistribution of free CPT-II (4 mg/kg) and CPT-I I/ONT (I00 mg ONT/kg, equivalent to 2 3.5 mg CPT-I I/kg) at 24 hours after a single intravenous injection into mice bearing $\mathrm{C} 26$ tumor. Tissue and tumor biodistribution of CPT-II (A) and SN-38 (B).

Notes: Each value represents the mean \pm SE $(n=3)$. $* P<0.05$.

Abbreviations: ONTs, organic nanotubes; SE, standard error.

water-soluble marker inside ONTs, and Gd-ONT, as an ONT marker, and compared with that of $3 \mu \mathrm{m}$ MP which was similar size to the length of ONTs. This study showed that similar to the distribution of Gd-ONT, CPT-11 loaded in ONTs and its active metabolite, SN-38, accumulated highly in the lung but less so in the liver and spleen. In contrast, $3 \mu \mathrm{m}$ MPs accumulated highly in the liver and spleen, but less so in the lung.
The loading amount of CPT-11 into ONTs increased as $\mathrm{pH}$ decreased. This might be related to the increased hydrophilicity and higher solubility of CPT-11 at lower pH caused by increased protonation of the tertiary amine group on CPT11. In terms of release, the release of CPT-11 from ONTs was higher at higher $\mathrm{pH}$. Unlike CPT-11, we reported previously that DXR was most efficiently loaded into ONTs at higher

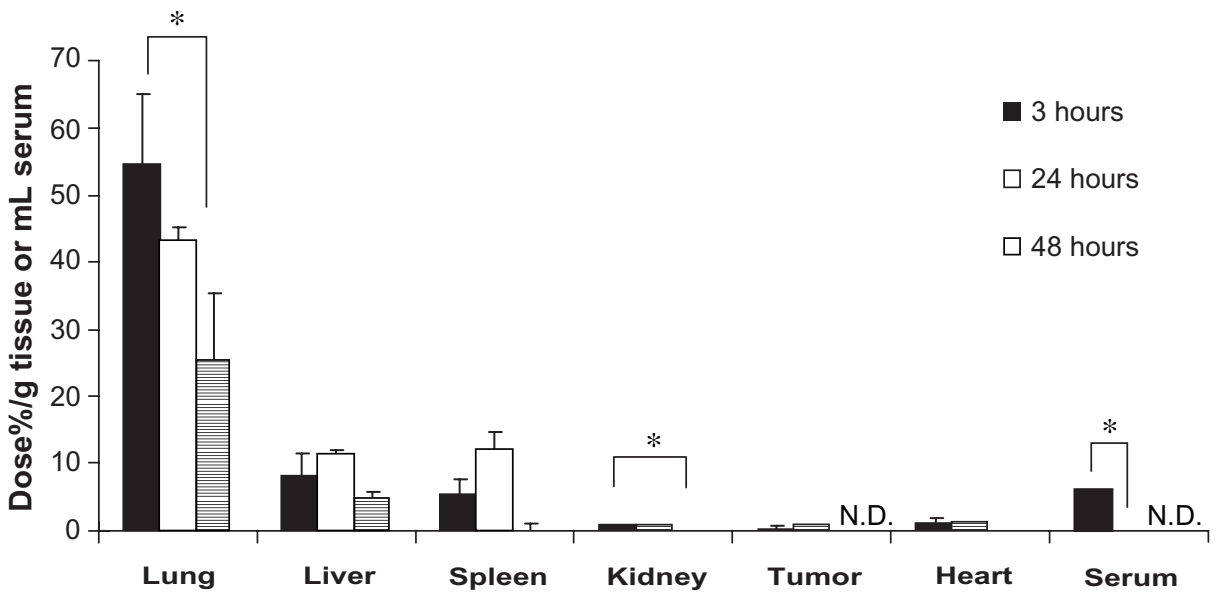

Figure 6 Biodistribution of Gd-ONT ( $50 \mathrm{mg} \mathrm{ONT/kg,} \mathrm{equivalent} \mathrm{to} 6.3 \mathrm{mg} \mathrm{Gd} / \mathrm{kg}$ ) at 3, 24, and 48 hours after a single intravenous injection into mice bearing C26 tumors. Notes: Each value represents the mean \pm SD $(n=3)$. $* P<0.05$.

Abbreviations: ONTs, organic nanotubes; SD, standard deviation. 


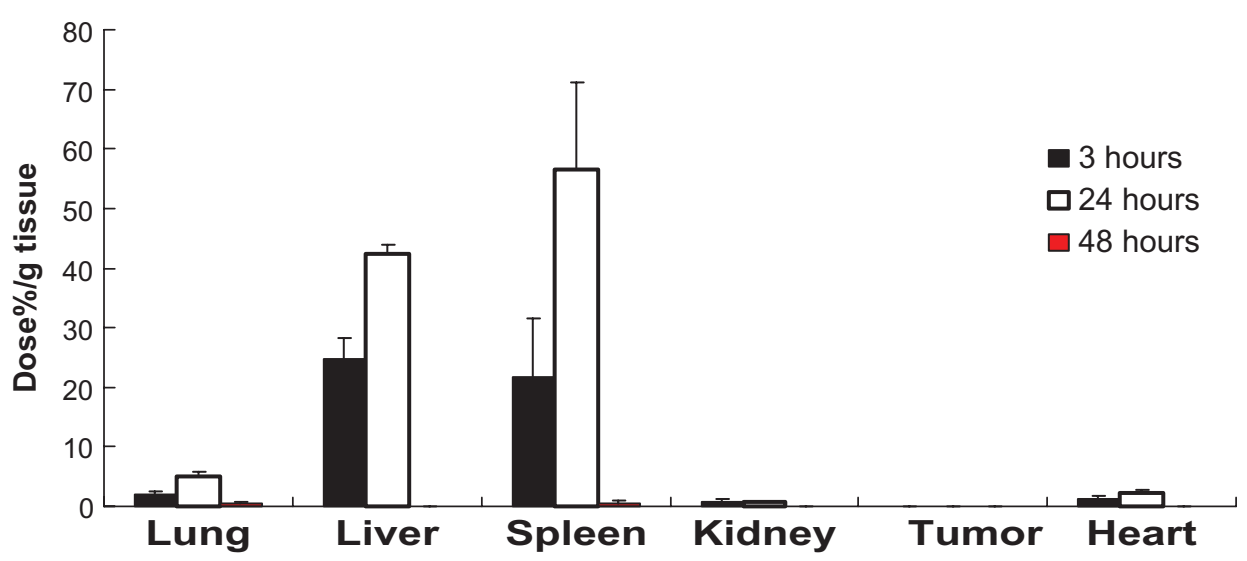

Figure 7 Biodistribution of MPs $(25 \mathrm{mg} / \mathrm{kg})$ at 3, 24 and 48 hours after a single intravenous injection into mice bearing C26 tumors. Notes: Each value represents the mean $\pm S D(n=3)$. $* P<0.05$.

Abbreviations: MPs, microparticles; ONTs, organic nanotubes; SD, standard deviation.

$\mathrm{pH}$, and DXR from ONTs was released less at higher $\mathrm{pH} .{ }^{11}$ Thus the $\mathrm{pH}$ condition for the optimal loading of drugs into ONTs was the opposite between CPT-11 and DXR. It was suggested that CPT-11 may interact more electrostatically with ONTs than DXR; therefore, the loading efficiency of CPT-11 (CPT-11: ONT = 1: 25, weight) was much lower than that of DXR (DXR: ONT = 1: 2, weight).

Because the different surface morphologies of particles affect the contact area with the epithelium in systemic circulation, their biodistribution is changed. Therefore, we tried to prepare large-sized spherical particles using
ONT lipid, compound 1, but we could not. Therefore, in this study we used spherical polystyrene MPs of size $3 \mu \mathrm{m}$. In the biodistribution of Gd-ONT, similar to CPT-11 in CPT-11/ONT, Gd-ONT accumulated highly in the lung ( $\sim 3 \mathrm{dose} \% / \mathrm{g})$, and less in the liver and spleen $(\sim 10 \mathrm{dose} \% / \mathrm{g})$ at 24 hours postinjection. Furthermore, CPT-11 and Gd-ONT were detected in the blood at 24 hours postinjection although the elimination half-life of CPT-11 and Gd-DTPA was 6 hours ${ }^{18}$ with a half-life in the order of 1 minute. ${ }^{17}$ However, it is notable that ONTs did not accumulate greatly in the liver and spleen like a sphere particle.

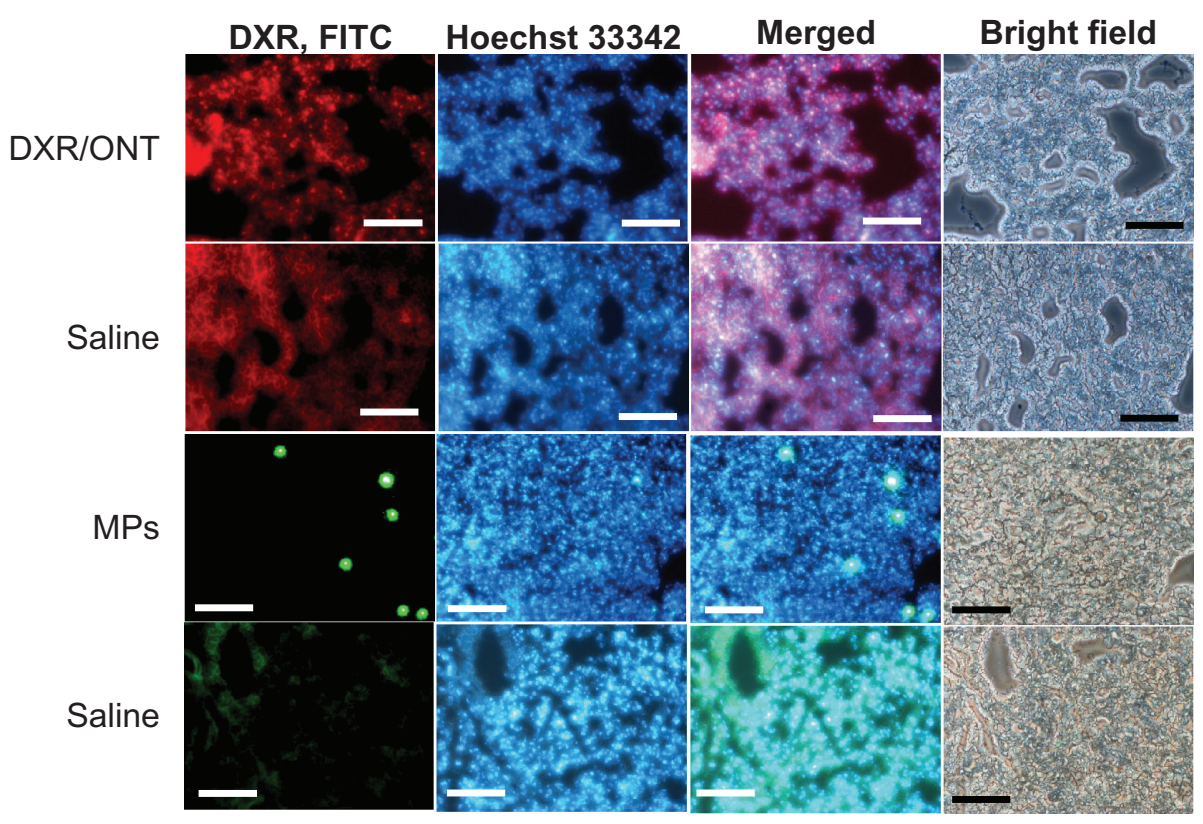

Figure 8 Biodistribution of DXR/ONT and MPs in lung tissues at 3 hours after a single intravenous injection into mice.

Notes: Fluorescence microscopy analyses confirming DXR/ONT, MPs and blood vessels (Hoechst 33342 staining) as red dots, green dots and blue fluorescence, respectively. Each saline was the control for red and green fluorescence. DXR/ONT was found outside of blood vessel in part, whereas MPs were found exclusively within the vessel lumen or associated with endothelial cells. Scale bars $=100 \mu \mathrm{m}$.

Abbreviations: DXR, doxorubicin; MPs, microparticles; ONTs, organic nanotubes. 

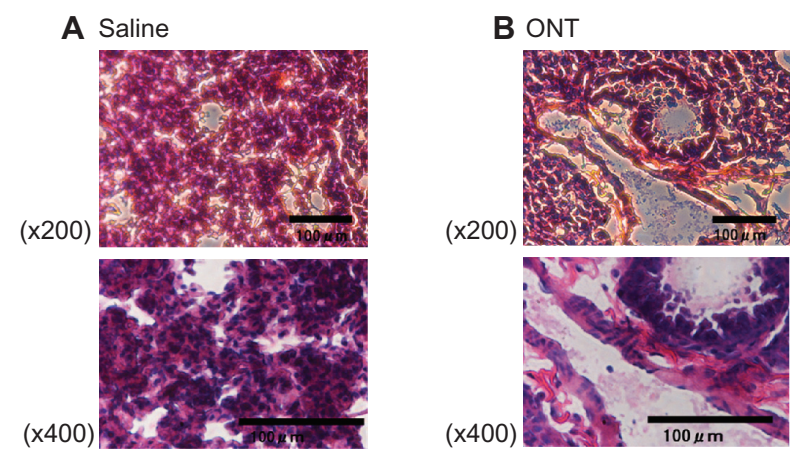

Figure 9 Hematoxylin and eosin-stained histological sections of lung tissue at 3 hours postinjection of saline $(\mathbf{A})$ or ONTs $(\mathbf{B})$.

Note: Scale bars $=100 \mu \mathrm{m}$.

Abbreviation: ONTs, organic nanotubes.

Even when the high accumulation of Gd-ONT in the lung decreased time-dependently, Gd-ONT accumulation in the liver and spleen was not increased correspondingly. This finding might be related to the report that macrophage internalization of worm-like particles of sizes 1 and $3 \mu \mathrm{m}$ was lower than spherical particles of the same size. ${ }^{19}$ The tube shape of ONTs might prevent ONTs from entering the liver and spleen by macrophages.

Lung accumulation of particles was reported for largesized MPs, cationic liposomes and immunoliposomes. Since the smallest capillaries of the lungs are especially relevant for MPs larger than 5-7 $\mu \mathrm{m}$ diameter in dogs and rats, $6 \mu \mathrm{m}$ MPs accumulated highly in the lung at 6 hours postinjection in rats. ${ }^{2,3}$ Smaller rigid particles $(<4 \mu \mathrm{m})$ pass through the lung and become entrapped in the liver and spleen. ${ }^{2,3}$ In contrast, silicon-based particles with discoidal shape and a diameter of $1.6 \mu \mathrm{m}$, have been observed to accumulate in the lung more than other shaped particles in most other organs. ${ }^{8}$ From this information, in neglecting morphological analyses, one of the factors may be shape rather than size to explain the high lung accumulation of ONTs with a length of approximately $2 \mu \mathrm{m}$.

Cationic liposomes complexed with plasmid DNA were reported to be highly accumulated in the lung when injected intravenously. ${ }^{20}$ The reason for the complex localization in the lung is related to agglutination caused by electrostatic interaction between positively charged complex and negatively charged erythrocytes. Interestingly in the present study, negatively charged ONTs of which zeta-potential was $-8 \mathrm{mV}$, accumulated largely in the lung.

Immunoliposomes are generated by conjugating antibodies to lipid bilayer of liposomes. Targeting to the pulmonary endothelium by immunoliposome was reported to be $14.3 \%$ of the dose in the lung at 2 hours postinjection. ${ }^{21}$ ONTs showed a distribution of $8.3 \%$ of the dose in the lung at 3 hours postinjecion, which was similar to the lung targeting of immunoliposomes.

Furthermore it was confirmed that DXR/ONT was detected in lung tissues outside of the blood vessels in part, whereas MPs were in blood vessels. MPs of $3 \mu \mathrm{m}$ diameter could not migrate from blood vessels into lung tissues, possibly as a result of their large size. This was consistent with the report that intravascular injection of $3.2 \mu \mathrm{m}$ diameter microbeads through the jugular vein were retained within narrow pulmonary capillaries in mice. ${ }^{22}$ On the other hand, ONTs could migrate into the lung tissue without a large change in lung tissue integrity from histological observations. This effect might be caused by a special internalization mechanism of tube-shaped particles inside cells. ${ }^{11,23,24}$ This finding corresponded with the active metabolite, SN38, which was also highly detected in the lung similar to CPT-11 postinjection of CPT-11/ONT. Thus higher deposition efficiency and prolonged retention of ONTs in the lung may translate into reduced doses and less frequent administration, which will open the way for the development of new lung-targeting carriers.

\section{Conclusion}

In this study, we examined the potential of ONTs as a drug carrier in vivo and demonstrated that the biodistribution of ONTs was quite different from that of $3 \mu \mathrm{m}$ MPs which was similar size to the length of ONT. Intravenously injected CPT-11/ONT and Gd-ONT exhibited higher accumulation of CPT-11 and Gd-ONT in the lung, but lower accumulation in the liver and spleen. This is the first report that a tube-shaped carrier with a length of $\sim 2 \mu \mathrm{m}$ is capable of effective biodistribution in the lung. However, for the application of ONTs as drug carriers, further in vitro evaluation of interactions with lung cells must be investigated before this approach can be applied in vivo.

\section{Acknowledgments}

We are grateful to Drs Masayuki Yokoyama and Kouichi Shiraishi of The Jikei University for ICP measurements. This study was supported in part by the Science Research Promotion Fund by the Promotion and Mutual Aid Corporation for Private Schools of Japan.

\section{Disclosure}

The authors report no conflicts of interest in this work. 


\section{References}

1. Awasthi VD, Garcia D, Goins BA, Phillips WT. Circulation and biodistribution profiles of long-circulating PEG-liposomes of various sizes in rabbits. Int J Pharm. 2003;253(1-2):121-132.

2. Slack JD, Kanke M, Simmons GH, DeLuca PP. Acute hemodynamic effects and blood pool kinetics of polystyrene microspheres following intravenous administration. J Pharm Sci. 1981;70(6):660-664.

3. Kutscher HL, Chao P, Deshmukh M, et al. Threshold size for optimal passive pulmonary targeting and retention of rigid microparticles in rats. J Control Release. 2010;143(1):31-37.

4. Wang JP, Maitani Y, Takayama K, Nagai T. Pharmacokinetics and antitumor effect of doxorubicin carried by stealth and remote loading proliposome. Pharm Res. 2000;17(7):782-787.

5. Tasciotti E, Liu X, Bhavane R, et al. Mesoporous silicon particles as a multistage delivery system for imaging and therapeutic applications. Nat Nanotechnol. 2008;3(3):151-157.

6. Al Faraj A, Fauvelle F, Luciani N, et al. In vivo biodistribution and biological impact of injected carbon nanotubes using magnetic resonance techniques. Int J Nanomedicine. 2011;6:351-361.

7. Geng Y, Dalhaimer P, Cai S, et al. Shape effects of filaments versus spherical particles in flow and drug delivery. Nat Nanotechnol. 2007;2(4):249-255.

8. Decuzzi P, Godin B, Tanaka T, et al. Size and shape effects in the biodistribution of intravascularly injected particles. J Control Release. 2010;141(3):320-327.

9. Shimizu T, Masuda M, Minamikawa H. Supramolecular nanotube architectures based on amphiphilic molecules. ChemInform. 2005;36(32):1401-1443.

10. Kogiso M, Zhou Y, Shimizu T. Instant preparation of self-assembled metal-complexed lipid nanotubes that act as templates to produce metal-oxide nanotubes. Adv Mater. 2007;19(2):242-246.

11. Wakasugi A, Asakawa M, Kogiso M, Shimizu T, Sato M, Maitani Y. Organic nanotubes for drug loading and cellular delivery. Int J Pharm. 2011;413(1-2):271-278.

12. Masaki K, Masaru A, Masumi A, Toshimi S. Semisolid phase synthesis of metal-complexed organic nanotubes. Chem Lett. 2010;39(8): $822-823$.
13. Hattori Y, Shi L, Ding W, et al. Novel irinotecan-loaded liposome using phytic acid with high therapeutic efficacy for colon tumors. J Control Release. 2009;136(1):30-37.

14. Iwase Y, Maitani Y. Octreotide-targeted liposomes loaded with CPT-11 enhanced cytotoxicity for the treatment of medullary thyroid carcinoma. Mol Pharm. 2011;8(2):330-337.

15. Fluorescent Microsphere Resource Center. Manual for Using Fluorescent Microspheres to Measure Regional Organ Perfusion: University of Washington, Division of Pulmonary and Critical Care Medicine; 1999. Available from http://fmrc.pulmcc.washington.edu/DOCUMENTS/ FMRCCover99.pdf. Accessed November 27, 2012.

16. Hattori Y, Ubukata H, Kawano K, Maitani Y. Angiotensin II-induced hypertension enhanced therapeutic efficacy of liposomal doxorubicin in tumor-bearing mice. Int J Pharm. 2011;403(1-2):178-184.

17. Ma H, Shiraishi K, Minowa T, et al. Accelerated blood clearance was not induced for a gadolinium-containing PEG-poly(L-lysine)-based polymeric micelle in mice. Pharm Res. 2010;27(2):296-302.

18. Baker JH, Lam J, Kyle AH, et al. Irinophore C, a novel nanoformulation of irinotecan, alters tumor vascular function and enhances the distribution of 5-fluorouracil and doxorubicin. Clin Cancer Res. 2008;14(22): $7260-7271$.

19. Champion JA, Mitragotri S. Shape induced inhibition of phagocytosis of polymer particles. Pharm Res. 2009;26(1):244-249.

20. Gjetting T, Arildsen NS, Christensen CL, et al. In vitro and in vivo effects of polyethylene glycol (PEG)-modified lipid in DOTAP/cholesterolmediated gene transfection. Int J Nanomedicine. 2010;5:371-383.

21. Maruyama K, Holmberg E, Kennel SJ, Klibanov A, Torchilin VP, Huang L. Characterization of in vivo immunoliposome targeting to pulmonary endothelium. J Pharm Sci. 1990;79(11):978-984.

22. Ikegami M, Weaver TE, Grant SN, Whitsett JA. Pulmonary surfactant surface tension influences alveolar capillary shape and oxygenation. Am J Respir Cell Mol Biol. 2009;41(4):433-439.

23. Doshi N, Mitragotri S. Needle-shaped polymeric particles induce transient disruption of cell membranes. J R Soc Interface. 2010;7 Suppl 4: S403-S410.

24. Gratton SE, Ropp PA, Pohlhaus PD, et al. The effect of particle design on cellular internalization pathways. Proc Natl Acad Sci USA. 2008;105(33):11613-11618.
International Journal of Nanomedicine

\section{Publish your work in this journal}

The International Journal of Nanomedicine is an international, peerreviewed journal focusing on the application of nanotechnology in diagnostics, therapeutics, and drug delivery systems throughout the biomedical field. This journal is indexed on PubMed Central,

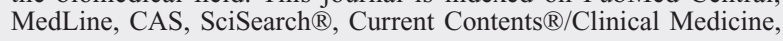

\section{Dovepress}

Journal Citation Reports/Science Edition, EMBase, Scopus and the Elsevier Bibliographic databases. The manuscript management system is completely online and includes a very quick and fair peer-review system, which is all easy to use. Visit http://www.dovepress.com/ testimonials.php to read real quotes from published authors. 